\title{
Proposal of Cooperative Communication to Enhance Accuracy of Wireless Control Systems
}

\author{
Nguyen Cong Dinh \\ Le Quy Don Technical University, Ha Noi, Vietnam \\ Email:dinhnc@mta.edu.vn
}

How to cite this paper: Dinh, N.C. (2019) Proposal of Cooperative Communication to Enhance Accuracy of Wireless Control Systems. Communications and Network, 11, 52-63.

https://doi.org/10.4236/cn.2019.112005

Received: February 19, 2019

Accepted: April 1, 2019

Published: April 4, 2019

Copyright $\odot 2019$ by author(s) and Scientific Research Publishing Inc. This work is licensed under the Creative Commons Attribution International License (CC BY 4.0).

http://creativecommons.org/licenses/by/4.0/

\section{cc) (i) Open Access}

\begin{abstract}
Control systems are being changed from wired to wireless communications because of flexibility, mobility and extensibility of wireless communication systems; however the reliability of wireless communications is suspected. In this paper, we propose cooperative communication scheme for wireless control systems which consist of a controller and multiple machines; these machines cooperatively work in a group and for the same duty. In the proposed method, the controller can communicate with machines directly or via other machines, whereas in the conventional method, the controller only communicates with machines directly. The simple 2-link arm plant is used to evaluate our proposed system, and the simulation results indicate that the proposed method is more accurate, and more stable than the conventional method.
\end{abstract}

\section{Keywords}

Wireless Control System, 2-Link Arm Plant, Cooperative Communication Scheme, Cooperative Operation of Machines

\section{Introduction}

In recent years, because of development of wireless communication systems, wireless communication systems have been applied widely in many fields, such as robot systems, vehicle networks, quality control systems in agriculture and so on. Thus, reliable wireless control technologies have been demanded strongly [1] [2], especially for controlling equipment in medical applications [3].

In control theory, the decision on output of system is uniquely determined by the given input so far. However, wireless communications are the stochastic systems in which the relation between input and its output is unsettled. Therefore, in order to apply wireless communication to control systems, it is necessary to 
propose a suitable control method. Thus, when applying the wireless communication instead of the wired communication in control systems, not only reliability of communication part, but combination of communication unit and control unit also should be considered [4] [5]. Especially, in case multiple equipments are simultaneously operating; the control part is complicated.

The conventional communication scheme in wireless control system is the controler which directly communicates with machines. The previous research focused on proposing new method for wireless control system in order to reduce latency, increase spead or save energy [6]. The multiple-hop wireless communication scheme was applied for wireless control system to guarantee the latency; however the link between nodes was assumed to be beyond-line-of-sight [7].

There are several researches on cooperative motion control system which proposed algorithms to control machines for cooperative operation [8] [9]. However, in these researches, the cooperative operation of machines was analyzed under assumption that communication between controller and machines, between machines is ensured. In fact, this assumption is no longer available because machines are moving and the communication between the controller and a machine, between machines is not always ensured. Therefore, in this paper, we discuss on wireless control systems for cooperation of machines under the imperfect communication between the controller and machines. Multiple 2-link arms plants are used as an example of cooperative operation in control system.

Compared to the wired communication, the wireless communication has many advantages, such as mobility, flexibility, extensibility and so on; however the reliability is lower due to noise, fading and interference. One of technologies to enhance the reliability of wireless communication is to improve diversity gain [10] [11]. Therefore, we propose cooperative communication scheme of machines as a method of obtaining the diversity gain. In case the communication between the controller and some machines is unavailable; the controller and these machines communicate via the other machines. The proposed scheme is analyzed and compared to the conventional method. It is expected to be available to improve the reliability of wireless control system.

This paper is organized as follows. Wireless control for cooperative system is briefly described in Sec. 2. In Sec. 3, the cooperative communication method is proposed and analyzed. The performance of the proposed method is compared with the conventional method in Sec. 4. Finally, conclusions are given in Sec. 5.

\section{Wireless Control for Cooperative System}

\subsection{System Model}

In many fields, plants should work instead of human resources. However, operation and mobility of one big plant are restricted in several scenarios, such as lifting a heavy thing, operating in small space and so on. Therefore, the cooperation of plants was proposed because of its flexibility, mobility and extendibility. Structure of cooperative systems is one of problems when the cooperation of 
plants is applied; the following three systems are the conventional cooperative systems.

- One plant moves based on self-position and its own course plan, other plants become the followers. This system is called the leader-follower system. When operation environments are almost unchanged, the leader-follower system is valid; however this system is unsuitable for dynamic operation environment.

- All of plants observe the environment while operating; however a plant which found out obstacles becomes a leader. This system is called the leader shift system, and suitable for unknown environment searching system. However, if the number of plants increases, information exchanged between plants increase and carry out congestion.

- The leader which can recognize situation is assigned from all of plants, and other plants become the followers and act according to the order of the leader. This system is called the super leader system. This system works well in almost operation environment; however it requires one super signal processing plant to lead the other and stable communications between the leader and the followers.

The system that consists of two or more 2-link arm plants working together, is taken into consideration. One of applications is that, these plants convey a thing, such as patients, equipment, and so on. Moreover, because of concentration of information of all plants on the controller, the super leader system is applied. The proposed system model is shown in Figure 1.

\subsection{Wireless Control for 2-Link Arm Plant}

In the feedback loop of a discrete time control system, we suppose that transmission of control information from a controller to plants and state information from plants to controller is carried out by wireless communications as shown in Figure 2.

Transmission error occurs stochastically on both feedforward and feedback wire-less communication, furthermore the error can be detected at receiver site by error detection codes. In addition, we assume a random model for radio communication channels, and packet loss of both control information and status

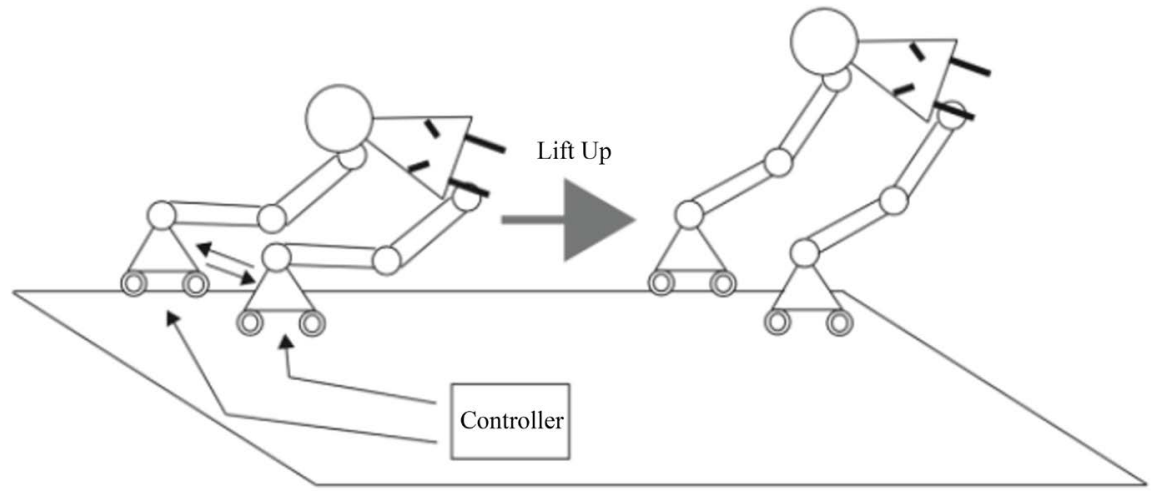

Figure 1. Cooperation of machines. 


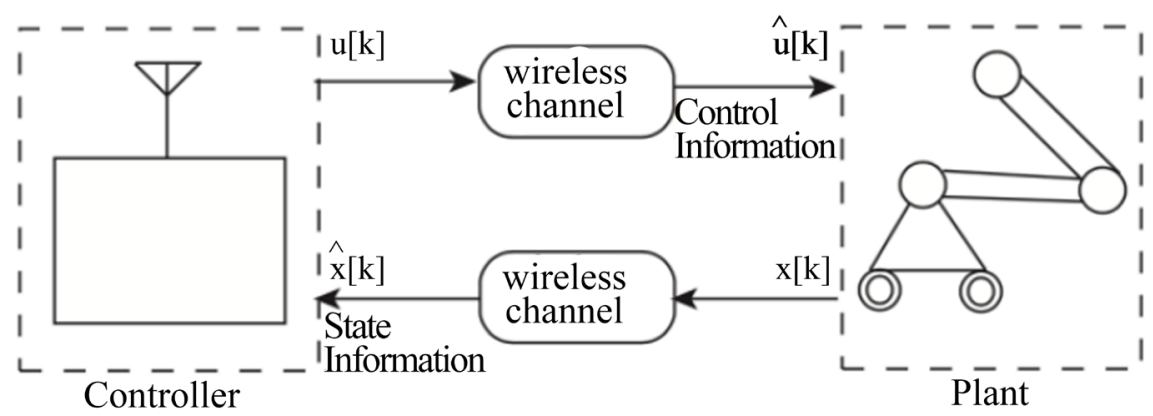

Figure 2. Concept of system.

information packets occurs with probability $p$. In time domain, when the error is detected, the previous errorless packet is used instead of.

$$
\begin{aligned}
& \hat{u}[k]=\left\{\begin{array}{l}
u[k], \text { without error } \\
u[k-1], \text { with error, }
\end{array}\right. \\
& \hat{x}[k]=\left\{\begin{array}{l}
x[k], \text { without error } \\
x[k-1], \text { with error, }
\end{array}\right.
\end{aligned}
$$

here $u[k]$ and $x[k]$ are respectively the data packet of control information and status information in $k^{\text {th }}$ time slot, while $\hat{u}$ and $\hat{x}$ are the estimated one at receiver site.

Simple diagram of 2-link arm which is a plant of wireless control systems is shown in Figure 3. Control information is sent from the controller to the plant, and the plant operates based on that information. Whereas, the state information of the plant is sent to the controller, and the controller processes that information.

Equation of motion of the 2-link arm is represented as follows [4].

$$
M(\mathbf{q}) \ddot{\mathbf{q}}+\mathbf{h}(\mathbf{q}, \dot{\mathbf{q}})+\mathbf{G}(\mathbf{q})=\tau,
$$

and it can be described in detail by

$$
\begin{aligned}
& {\left[\begin{array}{cc}
I_{1}+I_{2}+m_{2} l_{1}^{2}+2 m_{2} l_{1} r_{2} \cos \theta_{2} & I_{2}+m_{2} l_{1} r_{2} \cos \theta_{2} \\
I_{2}+m_{2} l_{1} r_{2} \cos \theta_{2} & I_{2}
\end{array}\right]\left[\begin{array}{l}
\ddot{\theta}_{1} \\
\ddot{\theta}_{2}
\end{array}\right]} \\
& +\left[\begin{array}{c}
-m_{2} l_{1} r_{2} \sin \left(\theta_{2}\left(2 \dot{\theta}_{1} \dot{\theta}_{2}+\dot{\theta}_{2}^{2}\right)\right) \\
m_{2} l_{1} r_{2} \sin \dot{\theta}_{2} \dot{\theta}_{1}
\end{array}\right]+\left[\begin{array}{c}
\left(m_{1} r_{1}+m_{2} r_{2}\right) \cos \theta_{1}+m_{2} r_{2} \cos \left(\theta_{1}+\theta_{2}\right) \\
m_{2} r_{2} \cos \left(\theta_{1}+\theta_{2}\right)
\end{array}\right] \mathbf{g} \\
& =\left[\begin{array}{c}
\tau_{1} \\
\tau_{2}
\end{array}\right]
\end{aligned}
$$

Here, $l_{i}, m_{i}, \theta_{i}$ denotes the length of the link between the axes, the mass of the link, the angle of rotation, respectively. $\tau_{i}$ is the torque generated by rotating shaft, $r_{i}$ is the distance between rotating shaft and the center of gravity, $I_{i}$ is the moment of inertia around the rotating shaft, and $\mathbf{g}$ is the acceleration of gravity to the negative direction of axis $\mathrm{z}$.

The first term in the left-hand side of (5) is the inertial force term. The second term represents centrifugal force and the Coriolis term. The third term is the gravity term. 


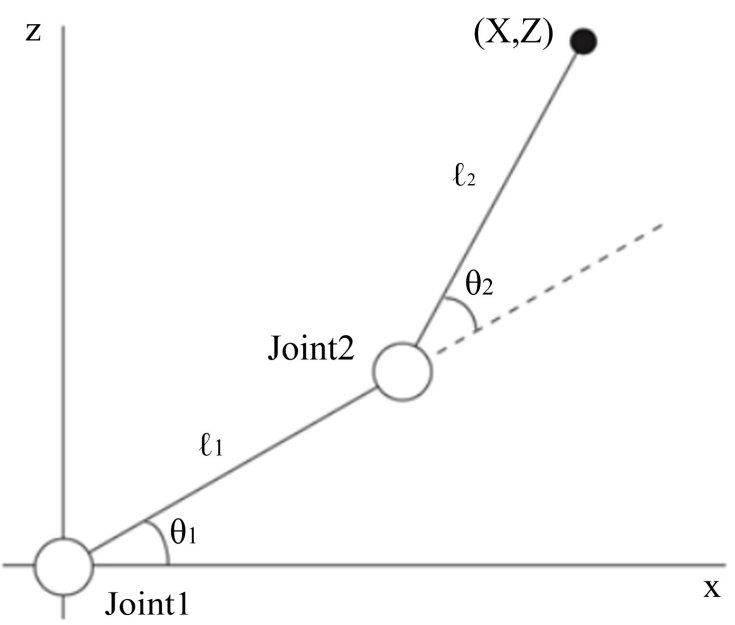

Figure 3. 2 link arm.

As explained above, the status information of plants is sent to the controller by feedback channel of wireless communication, and then the controller calculates the torque command value based on the status information and sends back to the plant. Because a proportional integral derivative controller (PID controller) is a control loop feedback mechanism widely used in industrial control systems and a variety of other applications requiring continuously modulated control, PID controller is used in this work. The calculation formula of torque information of PID controller is depicted as follows [12].

$$
\tau[k]=\tau[k-1]+K_{p}\left((e[k]-e[k-1])+\frac{\Delta t}{T_{I}} e[k]+\Delta d[k]\right),
$$

where

$$
\Delta d[k]=\frac{\eta T_{D}}{\Delta t+\eta T_{D}} \Delta d[k-1]+\frac{\eta T_{D}}{\Delta t+\eta T_{D}}(e[k]-e[k-1]+e[k-2]),
$$

and $\tau$ denotes torque information and $e$ denotes deviation of the state information of the plant. $K_{p}, T_{i}$ and $T_{d}$ respectively represents proportional gain, integration time and derivative time.

\section{Analysis of Cooperative Control System}

\subsection{Operation of System}

As shown in Figure 4, in the control system, first, the next point on orbit of the object is decided by the controller, and then the controller calculates the desired values for each plant according to present states and balance of power of plants. The position of balance is calculated from the correlation of plants and the object, furthermore, the controller calculates the torques by (5) and sends them to every plant in order. The controller retransmits the torque information in the case of packet loss until the number of retransmissions reaches the limitation in feedforward communication. The feedforward communication is finished when the number of retransmissions reaches the limitation or all of plants successfully 


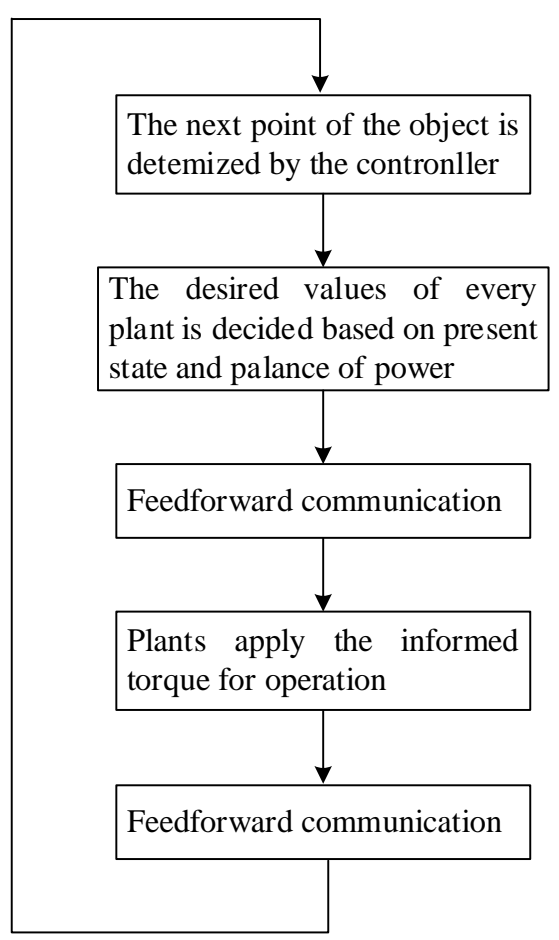

Figure 4. Flow chart of control system.

received the torque information. After completing the feedforward communication, the additional force is calculated by (6) based on the present state and inputted information.

$$
\tau_{e x}=\left[\begin{array}{cc}
-l_{1} \sin \theta_{1}-l_{2} \sin \left(\theta_{1}+\theta_{2}\right) & -l_{2} \sin \left(\theta_{1}+\theta_{2}\right) \\
l_{1} \cos \theta_{1}+l_{2} \cos \left(\theta_{1}+\theta_{2}\right) & l_{2} \cos \left(\theta_{1}+\theta_{2}\right)
\end{array}\right]^{T} \times \mathbf{F}_{\mathbf{e}}
$$

Therefore, the inputted torque of plants is calculated by

$$
M(\mathbf{q}) \ddot{\mathbf{q}}+\mathbf{h}(\mathbf{q}, \dot{\mathbf{q}})+\mathbf{G}(\mathbf{q})=\tau-\tau_{e x},
$$

Every plant operates by this inputted torque, and then transmits its own state information to the controller in feedback communication. Similar to the feedforward communication, if there is packet loss, the plant retransmits its own state information packet until the number of retransmissions reaches the limitation. The feedback communication is finished when the number of retransmissions reaches the limitation or the state information of all plants is successfully transmitted to the controller. We assume that the time division multiple access (TDMA) scheme is applied for both feedforward and feedback communications because of its simplicity and evitable collision.

\subsection{Proposed Communication Scheme}

In general cases of remote control cooperative system, the plants work nearby together, and the distance between the plants is almost closer than the distances from the plants to the controller. Therefore, communications between the plants are better than communications between the plants and the controller. Especial- 
ly, in whole of duty, the communications between several plants and the controller may be interrupted by obstacles. The loss of communications between several plants and the controller affects whole of system, the delay is occurred and the reliability of system is deteriorated. In order to keep stable communications between the plants and the controller, we propose to apply the communication between the plants into feedforward and feedback communications. The plants which could not receive the data packet of desired values, are named the hidden plants, whereas the other plants are named the exposed plants.

In the feedforward communication of our proposed communication scheme, when the controller does not receive the acknowledgement (ACK) from hidden plants to confirm the receipt of desired values, the controller retransmits the data packet of desired values for these plants. The exposed plants also can receive the retransmission data packet, and recognize that there are some hidden plants could not receive the information from the controller. Thus, the exposed plants act as a relay, forward the retransmission data packet to the hidden plants and also for-ward the ACK to the controller following the carrier sense multiple access with collision avoidance (CSMA/CA) scheme. Because a plant incidentally becomes the hidden plant or exposed plant, the time slot for forwarding the retransmission data packet could not be assigned. Therefore, the TDMA scheme is unsuitable, and the CSMA/CA scheme can avoid the transmission collision by choosing a random integer number for backoff counter, the CSMA/CA is suggested to apply. The feedforward communication is finished if the controller receives the ACK of all plants, or the number of retransmissions reaches the limitation, or the time for this sampling period is out.

Similarly, in the feedback communication of the proposed communication scheme, the hidden plants could not send the present state information to the controller, and they should retransmit the data packet of present state information. The exposed plants forward the retransmission data packet of present state information to the controller, and then forward the ACK of the controller to the hidden plants.

Thank to the help of exposed plants, the communication between the controller and the hidden plants becomes available. It let the delay reduce as well as the reliability of system increase. We analyze the proposed system and then compare to conventional system in the following section.

\section{Performance Evaluation}

\subsection{Scenario of Simulation}

The system parameters summarized in Table 1 are used to setup the system. The number of hidden plants in every sampling period is a random integer number within [1, N-1]; here $\mathrm{N}$ denotes the number of all plants, whereas the communication between plants is certain. We assume that all of the plants connect correctly with the object and are ready to convey. The plants lift the object up and down while move it on the orbit which is a circle with diameter of $0.7 \mathrm{~m}$ in the vertical plane. 
Table 1. System parameters.

\begin{tabular}{cc}
\hline Parameter & Value \\
\hline Length of Link 1 [m] & 0.5 \\
Weight of Link 1 [kg] & 2 \\
Length of Link 2 [m] & 0.5 \\
Weight of Link 2 [kg] & 2 \\
Weight of Object [kg] & 400 \\
Sampling Period of Control [s] & 0.1 \\
Proportional Gain of Joint 1 & 255 \\
Integral Gain of Joint 1 & 0.025 \\
Differential Gain of Joint 1 & 0.20 \\
Proportional Gain of Joint 2 & 75 \\
Integral Gain of Joint 2 & 0.025 \\
Differential Gain of Joint 2 & 0.15 \\
Coefficient of Friction & 0.5 \\
Acceleration of Gravity [m/s $\left.{ }^{2}\right]$ & 9.8 \\
Limitation of retransmission of feedforward communication & 6 \\
\hline retransmission of feedback communication & 6 \\
\hline
\end{tabular}

In case the hidden plants cannot receive the information data packet from the controller after limitation of retransmission due to the loss of connection, the hidden plants apply the information in the previous errorless packet for operation. The force impacted on the object is the sum of forces of all plants, therefore, the object may be lifted out of the orbit and the smallest distance from the object to the orbit is called the error distance (Figure 5). Furthermore, the maximal error is the maximal value of all error distances after the object is lifted one round of orbit.

In the conventional system, the communication between the controller and the plants is direct, and the communication between the plants is unavailable. Whereas, in the proposed system, the communication between the controller and the plants is direct or via another plant, the communication between the plants is always available.

\subsection{Numerical Results}

The maximal error distance when the object is moved with velocity of $1 \mathrm{~km} / \mathrm{h}$, is shown in Figure 6. The maximal error of both conventional and proposed systems is increased when the number of plants increases. However, the maximal error of conventional system is higher and increases more rapidly than that of proposed system.

The reasons are clearly explained from Figure 7 which depicts the average of maximal number of retransmissions in every sampling period. Because of unavailable communication between the plants, the number of retransmissions of 


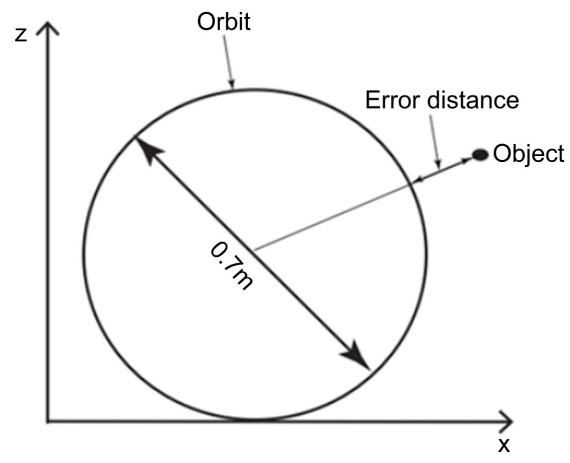

Figure 5. Orbit and error distance.

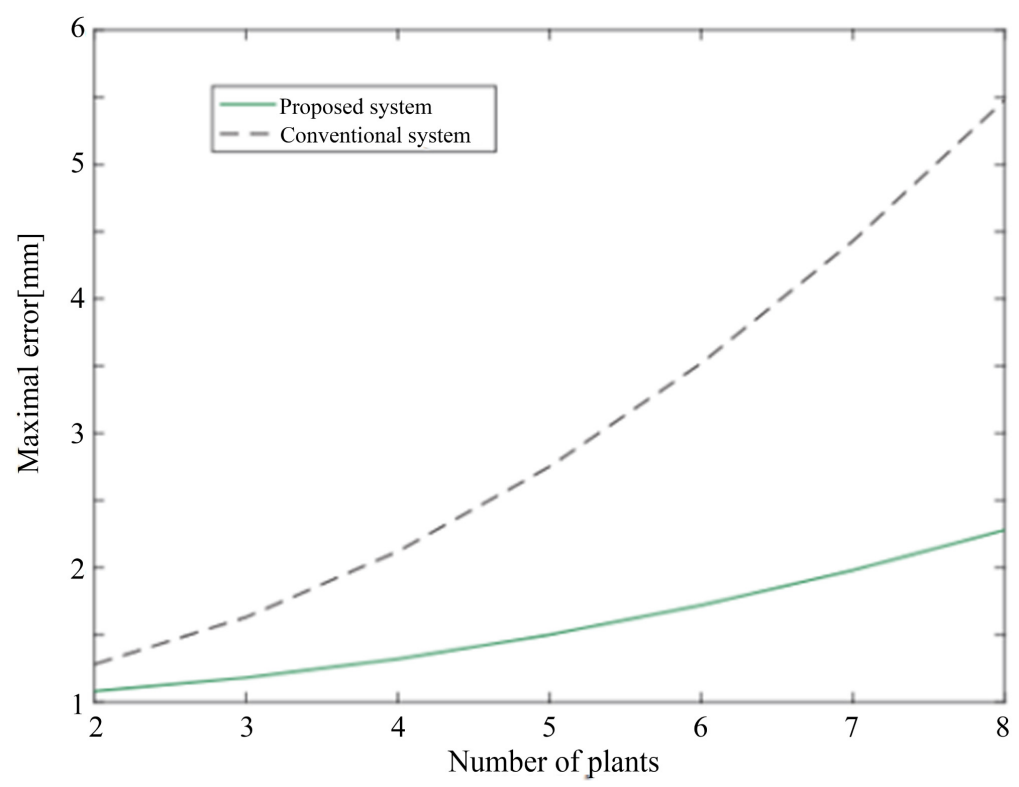

Figure 6. Maximal error distance in case of velocity of $1 \mathrm{~km} / \mathrm{h}$.

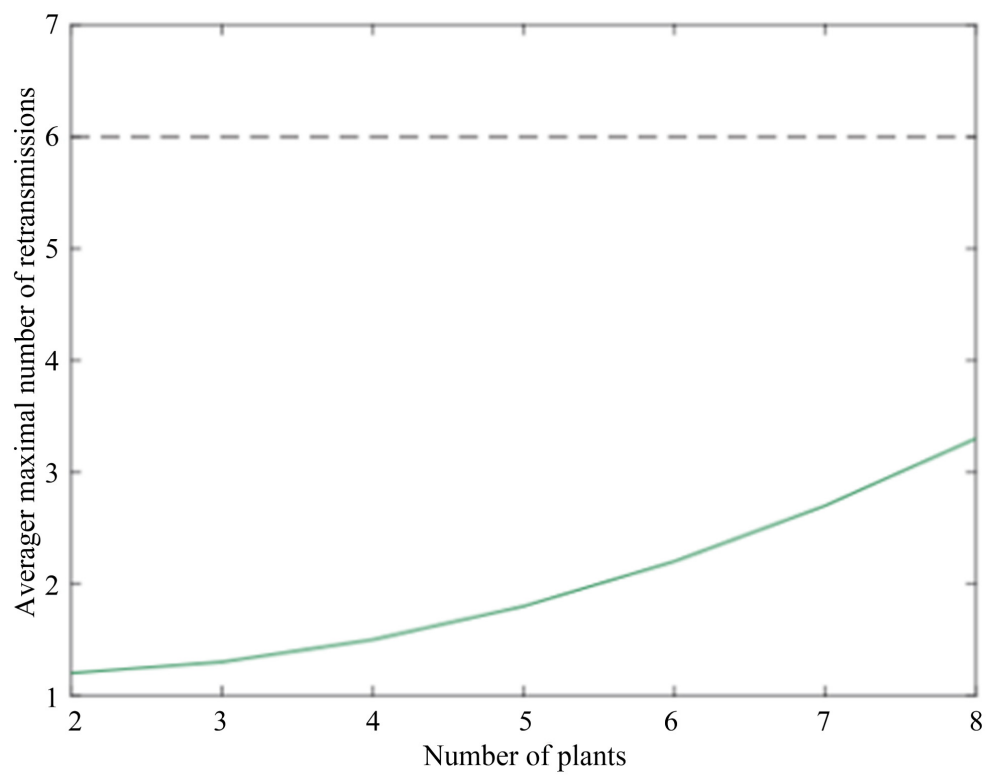

Figure 7. Average number of retransmissions. 
controller in the feedforward communication and the number of retransmissions of the hid-den plants in the feedback communication reach the limitation in every sampling period. Therefore, the average maximal number of retransmissions is also the maximal value, i.e. 6 .

However, in the proposed system, the communication between the plants is always available; therefore the controller and the hidden plants can communicate to each other via the exposed plants. Despite this, the communication between the controller and the hidden plants is not always available because of the communication error, the collision of data packets and the timeout. Especially when the number of hidden plants increases, the number of retransmissions as well as the number of unavailable communications between the controller and the hidden plants also increases. However, compared to the conventional system, in proposed system the number of hidden plants that finally could not communicate with the controller is much smaller, the maximal error is therefore considerably lower.

The performance of proposed system is also analyzed in case the velocity of object is twice, i.e. $2 \mathrm{~km} / \mathrm{h}$. As shown in Figure 8, the maximal error of both conventional and proposed systems is higher than that in Figure 6. However, compared to the case the velocity is a half (Figure 6); the maximal error of conventional in this case is much higher, whereas the maximal error of proposed system is slightly increased. It indicates that the communication of proposed system is more stable, and therefore the reliability of proposed system is much higher.

\section{Conclusions}

In this paper, the wireless control system with cooperative operation of multiple

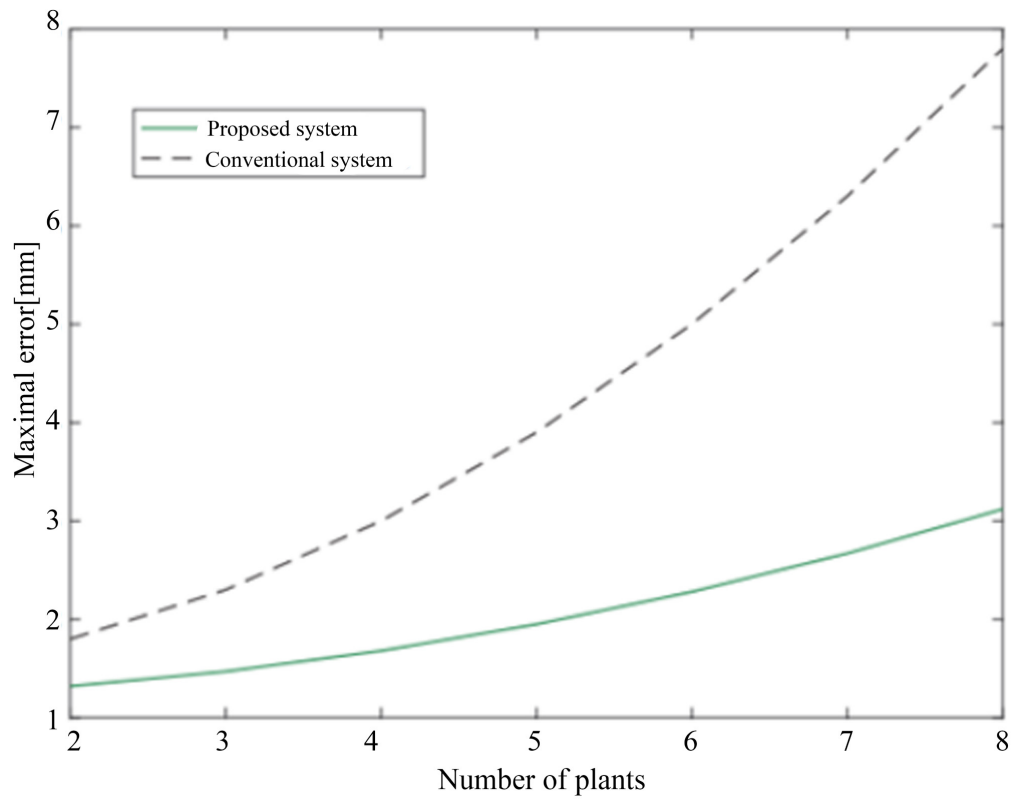

Figure 8. Maxima error in case of velocity of $2 \mathrm{~km} / \mathrm{h}$. 
machines was analyzed. The simple 2-link arm plants and circle of orbit were taken into consideration in order to evaluate the proposed system. In the proposed system, the cooperative communication scheme was offered; the controller can communicate with the plants directly or via the other plants, whereas in the conventional system the controller only communicates with the plants directly. The calculation result indicates that compared to the conventional system, the communication between the controller and the plants of proposed system is more certain; the maximal error is considerably lower. Furthermore, the proposed system is more stable even though the velocity of object increases.

In order to evaluate the proposed communication scheme, a simple system was taken into consideration. However, the system which has clear purpose and is similar to actual applications, will be used to evaluate the proposed communication scheme. Moreover, the control of transmission on MAC layer will be discussed to improve the performance of proposed method.

\section{Conflicts of Interest}

The author declares no conflicts of interest regarding the publication of this paper.

\section{References}

[1] Raymond, J.W., Olwal, T.O. and Kurien, A.M. (2018) Cooperative Communications in Machine to Machine (M2M): Solutions, Challenges and Future Work. IEEE Access, 6, 9750-9766. https://doi.org/10.1109/ACCESS.2018.2807583

[2] Mazumder, S. (2011) Wireless Networking Based Control. Springer, Berlin.

[3] Tanaka, T., Yamasuke, K., Goto, T., Sugimoto, T. and Kohno, R. (2010) Influence of Channel Errors on a Wireless-Controlled Insulin Pump. IEICE Technical Report, RRRC2010-16, 33-36.

[4] Kondo, T., Yamamoto, T. and Katayama, M. (2010) Effects and Mitigation of Channel Errors in a Wireless Control System for Cooperative Motion of Machines. IEICE Technical Report, WBS2010-11, 57-62.

[5] Nikolakopoulos, G. (2013) Lecture Notes on Industrial Automation and Process Control. Department of Computer Science Electrical and Space Engineering LTU, Sweden.

[6] Sadi, Y. and Ergen, S.C. (2017) Joint Optimization of Wireless Network Energy Consumption and Control System Performance in Wireless Networked Control Systems. IEEE Transactions on Wireless Communications, 16, 2235-2248. https://doi.org/10.1109/TWC.2017.2661280

[7] Kagawa, T., Ono, F., Shan, L., Takizawa, K., Miura, R., Kojima, F. and Kato, S. (2017) A Study on Latency-Guaranteed Multi-Hop Wireless Communication System for Control of Robots and Drones. 20th International Symposium on Wireless Personal Multimedia Communications (WPMC), Bali, 17-20 December 2017. https://doi.org/10.1109/WPMC.2017.8301849

[8] Kondo, T., Kobayashi, K. and Katayama, M. (2010) Mutual Use of Control Signals for Performance Improvement of a Wireless Cooperative Motion Control System. IEICE Technical Report, RRRC2010-14, 27-32.

[9] Park, P., Araujo, J. and Johanson, K. (2011) Wireless Networked Control System: 
Co-Design. International Conference on Networking Sensing and Control Netherland, Delft, 11-13 April 2011. https://doi.org/10.1109/ICNSC.2011.5874926

[10] Nosratinia, A., Hunter, T.E. and Hedayat, A. (2004) Cooperative Communication in Wireless Networks. IEEE Communication Magazine, 42, 74-80. https://doi.org/10.1109/MCOM.2004.1341264

[11] Sendonaris, A., Erkip, E. and Aazhang, B. (2003) User Cooperation Diversity. IEEE Transactions on Wireless Communications, 51, 1927-1947. https://doi.org/10.1109/TCOMM.2003.818096

[12] King, M. (2010) Process Control: A Practical Approach. John Wiley \& Sons Ltd., Hoboken. 